\title{
Impact of IDH1 Mutation on Long-Term Survival in Patients with Diffuse Glioma
}

\author{
Enkhee Ochirjav'1, Tuul Baldandorj', Gheeyoung Choe'², Bayarmaa Enkhbat ${ }^{1}$
}

'Department of Pathology, School of Biomedicine, Mongolian National University of Medical Sciences, Ulaanbaatar, Mongolia; ${ }^{2}$ Department of Pathology, Seoul National University Bundang Hospital, Seoul National University, College of Medicine, Seongnam, Korea

Submitted: February 1, 2019

Revised: February 21, 2019

Accepted: March 07, 2019

Corresponding Author

Enkhee Ochirjav, MD, MSc

Department of Pathology, School of

Biomedicine, Mongolian National

University of Medical Sciences,

Ulaanbaatar 14210, Mongolia

Tel: $+976-8901-2852$

Fax: + 62274563312

E-mail: enhee002@gmail.com

This is an Open Access article distributed under the terms of the Creative Commons Attribution Non-Commercial License (http:// creativecommons.org/licenses/bync/4.0/) which permits unrestricted non-commercial use, distribution, and reproduction in any medium, provided the original work is properly cited. Copyright $\odot 2019$ Mongolian National University of Medical Sciences
Objectives: Diffuse brain gliomas are common primary brain tumor associated with a poor prognosis. In this study, we aimed to determine the impact of IDH1 mutation status on long term survival. Methods: Patients who underwent surgery for diffuse brain glioma were selected. Based on IDH1 mutation status, patients were separated into IDH1 mutant and IDH1 wildtype groups. Results: A total of 124 patients with diffuse brain tumor were included (mean age $39 \pm 17,48.4 \%$ male). The frequency of IDH1 mutant and IDH1 wildtype were $56.5 \%$ $(n=70)$ and $43.5 \%(n=54)$, respectively. During follow-up, 76 patients $(61.3 \%)$ died and the median follow-up was 8 months (IQR 4; 16). Patients with IDH1 mutant more likely younger ( $36 \pm 16$ vs. $43 \pm 17, p<.05$ ) and had low grade (grade II) tumor (64.3\% vs. $20.4 \%, p<.001$ ). After adjustment of possible predictors, such age, gender, tumor location and surgical type, IDH1 mutant was an independent predictor of all-cause mortality $(\mathrm{HR}=0.43,95 \% \mathrm{Cl} 0.26$ $0.71, p<.001)$. Kaplan-Meier estimation showed IDH1 mutant is associated with longer survival compared with IDH1 wildtype in low grade tumor (log rank $p<.001)$. Conclusion: Mutated IDH1 is an independent predictor of all-cause mortality in patients with diffuse brain glioma and resulted in longer survival compared with IDH1 wildtype.

Keywords: Glioma, Isocitrate Dehydrogenase, Prognosis, Survival

\section{Introduction}

Diffuse brain gliomas are most common type of primary brain tumors and they have variety of histologic subtypes. According to the histopathological and clinical manifestations established by the World Health Organization (WHO), gliomas are classified from grade I to grade IV'. Grade I gliomas are often considered to be benign, curable by complete surgical resection and have a good prognosis while grade II and III gliomas are malignant and capable of progression into invasive grade IV glioma having a poor prognosis 2,3 .

The progression of primary brain tumor is dependent upon several genes such as tumor protein 53 (TP53), phosphatase and tensin homolog (PTEN), epidermal growth factor receptor $(E G F R)^{4-7}$. The development of glioma tumor is sequential process and occurrence of above-mentioned gene mutations might be crucial for the transition from mild to aggressive form. For example, TP53 mutation occurs in the early stage of glioma tumors while PTEN mutation and an increase of EGFR is prominent in high grade tumors ${ }^{8-10}$. 
In high grade gliomas, mutations of the isocitrate dehydrogenase 1 (IDH1) gene are found in 70\% of grade II and III astrocytomas and oligodendrogliomas and in secondary glioblastomas which arise from low grade gliomas ${ }^{11}$. Conversely, primary glioblastomas develop de novo without IDH1 gene mutation, and progress rapidly progression and have worse prognosis $^{12}$. This has led to the hypothesis that IDH1 gene mutation has an important role in development of high-grade gliomas.

The IDH1 gene mutation can be detected by immunohistochemical (IHC) staining of the IDH1 mutant protein. The IHC staining method has several advantages. It is easily implemented in standard pathological laboratories, is cost effective and provides a way to evaluate morphologic expression of mutated protein ${ }^{13,14}$. The most frequent mutation which found in up to $95 \%$ of glioma cases is a substitution of amino acid arginine by histidine at the codon $132(\mathrm{R} 132 \mathrm{H})^{15}$. There are two monoclonal antibodies that can be used for anti-IDH1-R132H immunohistochemical analysis with good concordance with DNA sequencing method ${ }^{14,16,17}$. Capper et al. demonstrated that IHC staining using mouse monoclonal anti-mIDHR ${ }^{132 H}$ antibody has 100\% sensitivity and specificity to detect R132H mutation, while sensitivity and specificity to detect all type of IDH1 mutation including R132G, R132S and R132C were 94\% and $100 \%$, respectively ${ }^{16}$.

In this study, we sought to determine the impact of IDH1 mutant protein on long term survival of patients with diffuse brain glioma.

\section{Material and Methods}

\section{Study design}

In this retrospective study, we evaluated tissue specimens from patients who underwent brain glioma removal surgery. Prior to beginning the study, the study design and ethics were approved by Institutional Review Board of Mongolian National University of Medical Sciences (№2017/3-201702, 17 February 2017). Data about age, gender, risk factors, previous comorbidities, laboratory measurements and surgical reports were retrospectively collected from patients' medical record. All the tumors were graded according to the WHO criteria and classified into high and low grade tumor'.

\section{Study population}

In this study, a total of 124 formalin fixed, paraffin embedded brain tumor tissue microarray (TMA) blocks which were collected and archived between 2006 and 2017 in the National Center for Pathology. The study population included tissue specimens collected from patients who had been diagnosed with diffuse glioma (WHO grade II-IV) confirmed by histological examination. Patients with WHO grade I glioma such as pilocytic astrocytoma and those whose tissue blocks were lost or too small were excluded.

Representative tumor areas were marked on hematoxylin and eosin (H\&E) stained sections. Tumor areas needed to contain over $60 \%$ tumor cell infiltrations and no necrosis or hemorrhage. Corresponding areas were identified on the paraffin blocks and tissue microarray blocks constructed and new $4 \mu \mathrm{m}$ thick sections were prepared.

\section{Immunohistochemical staining}

In this study, we used IHC staining method to reveal IDH1 $\mathrm{R} 132 \mathrm{H}$ mutation using mouse monoclonal anti-mIDHR ${ }^{132 \mathrm{H}}$ antibody (1:10 dilution, DIANOVA, Hamburg, Germany). For IHC staining, $4 \mu \mathrm{m}$ thick tissue sections were deparaffinized in xylene and hydrated by immersing in a series of graded ethanol baths. Antigen retrieval was performed in a microwave by placing the sections in epitope retrieval solution $(0.01 \mathrm{M}$ citrate buffer, pH 6.0) for 20 minutes; endogenous peroxidase was inhibited by immersing the sections in $0.3 \%$ hydrogen peroxide for 10 minutes. Sections were then incubated with IDH1 antibodies. Next, an Ultra View universal DAB kit was used following the manufacturer's recommendations (VENTANA, Tucson, Arizona, USA) in conjunction with an automated staining procedure. Finally, the samples were counterstained with haematoxylin, dehydrated, mounted, evaluated and photographed (20x and $40 \times$ objective) under a light microscope equipped with an Olympus CX21 camera. The immunoreactivity to mIDH1R132H antibody was evaluated for positive cytoplasmic or nuclear brown staining (Figure 1) 18,19. Based on IDH1 mutation status, patients were separated into IDH1 mutant (IDH1 ${ }^{\text {mut }}$ ) and IDH1 wildtype (IDH $1^{\text {wt }}$ groups.

\section{Study endpoint}

In this study, we choose all-cause mortality after hospital discharge for primary endpoint. Data on the occurrence of 


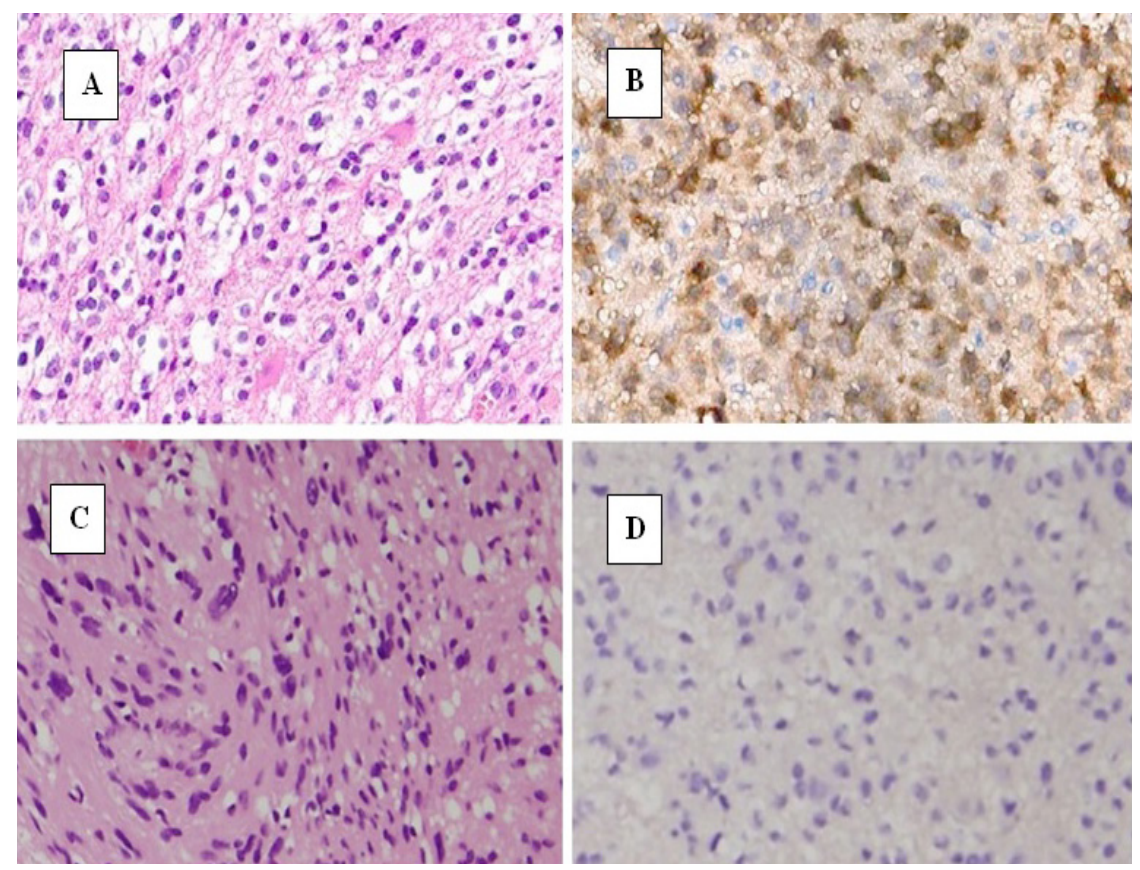

Figure 1. IDH1 immunohistochemical marker staining of diffuse brain glioma. (A) Definite fried egg appearance of oligodendroglioma (arrow) in H\&E staining x100. (B) The tumor cells stained positive for IDH1 immunostaining in the cytoplasm (white arrow), and also in the nucleus (black arrow). The unstained cells in the brain parenchyma represent non neoplastic cells. (C) H\&E staining of anaplastic astrocytoma with spindle shape, hyperchromatic nucleus (arrow) x100. (D) No staining of IDH1 marker in tumor cell cytoplasm or nucleus.

endpoint was collected from the database of General Authority for State Registration.

\section{Statistical analysis}

Continuous variables that were normally distributed (as evaluated by Kolmogorov-Smirnov tests) were presented as the mean \pm standard deviation (SD) and categorical data were presented as frequencies and percentages. Differences in baseline characteristics between patients with and without IDH1 mutation were compared using the independent sample t-test and chi-squared test.

Separate univariate and multivariate Cox proportional hazard regression analysis was used to determine the relationship between possible predictors and mortality. Variables were selected for univariate Cox proportional hazard regression oneby-one. Those variables identified as significant in the univariate analysis were used in multivariate analysis to evaluate their independent association between IDH1 mutation and all-cause mortality. Variables including age, gender, tumor location, surgery type and IDH1 mutation were simultaneously compared using multivariate Cox proportional hazard regression. Additionally, Kaplan-Meier estimation was used to evaluate association between IDH1 mutation and long-term survival.

All statistical tests were two-sided, and $p<.05$ was considered statistically significant. The statistical analyses were performed using SPSS software (version 22.0, SPSS Inc., Chicago, IL, USA).

\section{Results}

\section{Baseline characteristics}

The mean age of diagnosis was $39 \pm 17$ years old and the prevalence of diffuse brain glioma was equal in both genders (male $48.4 \%$ and female $51.6 \%$ ). Prevalence of WHO grade II, grade III and grade IV tumors were $45.2 \%(n=56), 26.6 \%$ $(n=33)$ and $28.2 \%(n=35)$, respectively.

Based on IDH1 mutation status, 70 patients (56.5\%) were IDH $1^{\text {mut }}$ and $54(43.5 \%)$ were IDH $1^{\text {wt }}$. Patients with IDH $1^{\text {mut }}$ were more likely to be younger compared with patients with IDH1wt (36 \pm 16 vs. $43 \pm 17, p<.05$ ). Occurrence of IDH1 mutation was 
not different between male and female genders $(47.1 \%$ vs. $52.9 \%, p=.752)$.

The WHO tumor grade was less aggressive in IDH1 ${ }^{\text {mut }}$ group (grade II tumor $64.3 \%$, grade III tumor $35.7 \%$ and grade IV tumor $0 \%$ ) compared with IDH1 ${ }^{\text {wt }}$ group (grade II tumor 20.4\%, grade III tumor $14.8 \%$ and grade IV tumor $64.8 \%$ ) and this difference was statistically significant $(p<.001)$. Using the WHO tumor grade, all the tumors were classified into low grade (WHO grade II) and high grade (WHO grade III and grade IV). Patients with IDH $1^{\text {mut }}$ were more likely had low grade tumor than patients with IDH1 ${ }^{\text {wt }}$ (64.3\% vs. $20.4 \%, p<.001$ ).

There was no statistically significant difference in tumor location between patients with and without IDH1 mutation $(p=.247)$. There was a trend towards a higher percentage of complete tumor removal surgery in the IDH1mut group than in IDH1 $1^{\text {wt }}$ group (54.3\% vs. $\left.37 \%, p=.056\right)$. The baseline characteristics of study population were summarized in Table 1.

\section{IDH1 mutation and long-term survival}

The median survival time was 8 months (interquartile range [IQR] $4 ; 16)$ for study population and all-cause mortality occurred in 76 patients (61.3\%). The frequency of IDH $1^{\text {mut }}$ and IDH $1^{\text {wt }}$ was $56.5 \% \quad(n=70)$ and $43.5 \% \quad(n=54)$, respectively. Univariate Cox proportional hazard regression analysis was showed that IDH1 ${ }^{\text {mut }}(\mathrm{HR}=0.42,95 \% \mathrm{Cl} 0.26-0.68, \mathrm{p}<.001)$ and complete tumor removal $(H R=0.53,95 \% \mathrm{Cl} 0.33-0.85, p<.01)$ were significant predictor of all-cause mortality. Multivariate analysis which considered age, gender, tumor location, surgical type and IDH1 mutation status revealed that IDH1 ${ }^{\text {mut }}(\mathrm{HR}=0.43,95 \% \mathrm{Cl}$ 0.26-0.71, $p<.001)$ and complete tumor removal $(H R=0.60$, $95 \% \mathrm{Cl} 0.37-0.98, \mathrm{p}<.05)$ were independent predictors of allcause mortality (Table 2).

Table 1. Baseline characteristics.

\begin{tabular}{|c|c|c|c|c|}
\hline Variables & All patient $(n=124)$ & $\begin{array}{l}\text { Patients with IDH1 }{ }^{\text {mut }} \\
\qquad(\mathrm{n}=70)\end{array}$ & $\begin{array}{l}\text { Patients with IDH1wt } \\
\qquad(\mathrm{n}=54)\end{array}$ & $\mathrm{p}$-value \\
\hline Age & $39 \pm 17$ & $36 \pm 16$ & $43 \pm 17$ & $.025^{\mathrm{a}}$ \\
\hline Gender & & & & $.752^{b}$ \\
\hline male & $60(48.4 \%)$ & $33(47.1 \%)$ & $27(50 \%)$ & \\
\hline female & $64(51.6 \%)$ & 37 (52.9\%) & $27(50 \%)$ & \\
\hline WHO tumor grade & & & & $.001^{\mathrm{b}}$ \\
\hline Grade II & $56(45.2 \%)$ & $45(64.3 \%)$ & $11(20.4 \%)$ & \\
\hline Grade III & $33(26.6 \%)$ & $25(35.7 \%)$ & $8(14.8 \%)$ & \\
\hline Grade IV & $35(28.2 \%)$ & $0(0 \%)$ & $35(64.8 \%)$ & \\
\hline Tumor grade & & & & $.001^{\mathrm{b}}$ \\
\hline Low grade & $56(45.2 \%)$ & $45(64.3 \%)$ & $11(20.4 \%)$ & \\
\hline High grade & $68(54.8 \%)$ & $25(35.7 \%)$ & $43(79.6 \%)$ & \\
\hline Tumor location & & & & $.247^{b}$ \\
\hline temporal & $17(13.7 \%)$ & $6(8.6 \%)$ & $11(20.4 \%)$ & \\
\hline multiple & $37(29.8 \%)$ & $23(32.9 \%)$ & $14(25.9 \%)$ & \\
\hline frontal & $34(27.4 \%)$ & $20(28.6 \%)$ & $14(25.9 \%)$ & \\
\hline talamus & $13(10.5 \%)$ & $6(8.6 \%)$ & $7(13 \%)$ & \\
\hline cerebellum & $7(5.6 \%)$ & $6(8.6 \%)$ & $1(1.9 \%)$ & \\
\hline parietal & $16(12.9 \%)$ & $9(12.9 \%)$ & $7(13 \%)$ & \\
\hline Surgery type & & & & $.056^{\mathrm{b}}$ \\
\hline complete removal & $58(46.8 \%)$ & $38(54.3 \%)$ & $20(37 \%)$ & \\
\hline partial removal & $66(53.2 \%)$ & $32(45.7 \%)$ & $34(63 \%)$ & \\
\hline
\end{tabular}

aindependent sample t-test, ${ }^{b}$ Chi-Square test.WHO, World Health Organization; IDH1 ${ }^{\text {mut }}$, isocitrate dehydrogenase-1 mutated; IDH1 ${ }^{\text {wt }}$, isocitrate dehydrogenase-1 wild type. 
Table 2. Univariate and multivariate Cox proportional hazard regression for all-cause mortality.

\begin{tabular}{|c|c|c|c|c|c|c|}
\hline \multirow{2}{*}{ Variables } & \multicolumn{3}{|c|}{ Univariate analysis } & \multicolumn{3}{|c|}{ Multivariate analysis } \\
\hline & HR & $95 \% \mathrm{Cl}$ & $\mathrm{p}$-value & HR & $95 \% \mathrm{Cl}$ & $\mathrm{p}$-value \\
\hline Age & 0.99 & $0.98-1.01$ & .737 & & & \\
\hline Gender & 0.76 & $0.48-1.20$ & .238 & & & \\
\hline Tumor location & 1.08 & $0.93-1.25$ & .319 & & & \\
\hline Complete tumor removal & 0.53 & $0.33-0.85$ & .009 & 0.60 & $0.37-0.98$ & .04 \\
\hline IDH $1^{\text {mut }}$ & 0.42 & $0.26-0.68$ & .001 & 0.43 & $0.26-0.71$ & .001 \\
\hline
\end{tabular}

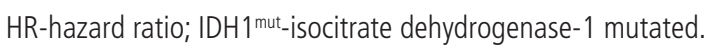

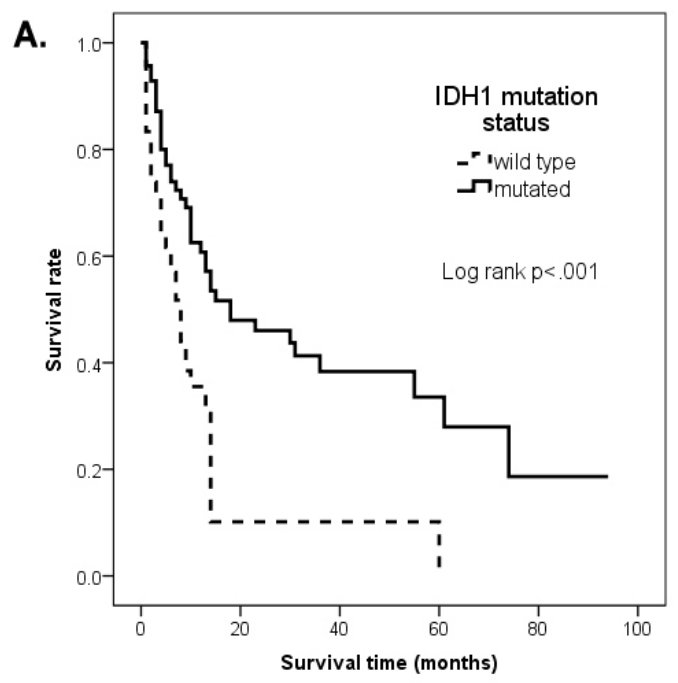

B.

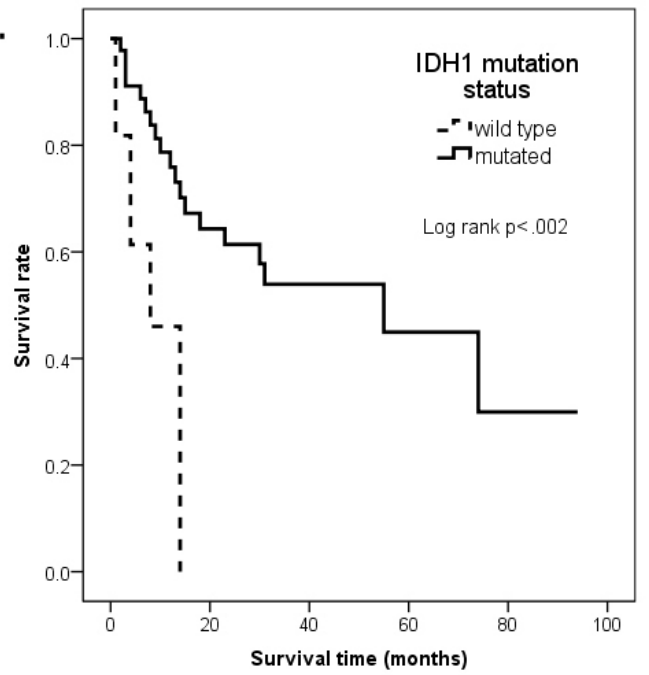

C.

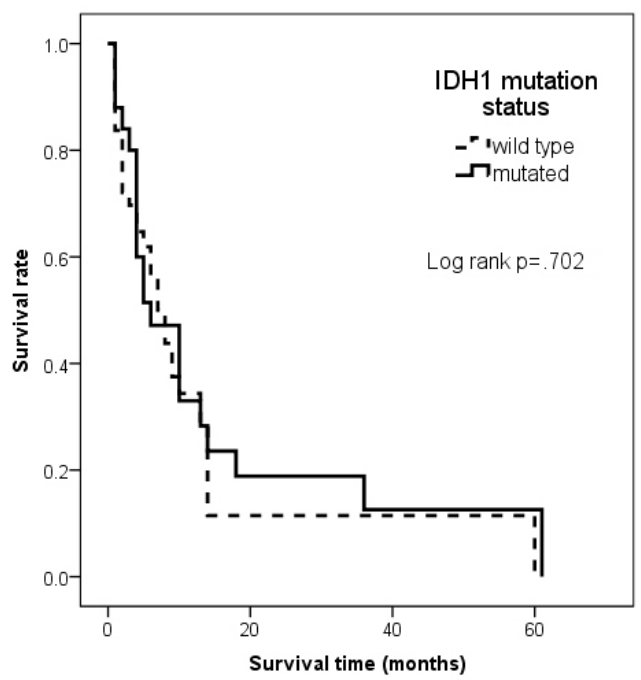

Figure 2. Kaplan-Meier estimation for survival rate. (A) all patients, (B) patients with low grade tumors, (C) patients with high grade tumors. IDH1 ${ }^{\text {mut }}$, isocitrate dehydrogenase-1 mutated; IDH1 ${ }^{\mathrm{wt}}$, isocitrate dehydrogenase-1 wild type.

Furthermore, Kaplan-Meier estimation was revealed that patients with IDH1 ${ }^{\text {mut }}$ (median survival 18 months, $95 \% \mathrm{Cl} 2-34$ months) were had longer survival compared with patients with IDH1wt (median survival 8 months, 95\% Cl 6-10 months, log rank $\mathrm{p}<.001)$. However, the association was only observed for low grade tumors (IDH1 mut median survival 55 months $95 \%$ Cl 13-96 months vs. IDH1 ${ }^{\text {wt }}$ median survival 8 months $95 \% \mathrm{Cl}$ 2-14 months, log rank $p=.002$ ). For patients with high grade 
tumors, IDH1 ${ }^{\text {mut }}$ didn't show survival benefit compared with IDH $1^{\text {wt }}$ (IDH1 ${ }^{\text {mut }}$ median survival 6 months 95\% CI 2-10 months vs. IDH1 $1^{\text {wt }}$ median survival 8 months $95 \% \mathrm{Cl} 5-10$ months, log rank $p=$.702) (Figure 2 ).

\section{Discussion}

In our study, we determined that $\mathrm{IDH}^{\text {mut }}$ is independent prognostic marker of all-cause mortality $(\mathrm{HR}=0.43,95 \% \mathrm{Cl}$ 0.26-0.71, $p<.001)$. Also, Kaplan-Meier curve estimation showed that patients with IDH1 ${ }^{\text {mut }}$ were had longer survival compared with IDH1wt. However, association was only evident for low grade tumors (grade II) $(p<.001)$. For patients with high grade tumors (grade III and IV), there was no long-term survival difference between IDH1 mutant and wild-type IDH1 ( $p=.702)$. These results are consistent with findings of Akagi et al. and Tabouret et al20,21.

Labussiere et al. determined that IDH1 gene mutation is relatively early event in pathogenesis of glioma and LV et al. noted that patients with IDH1 gene mutation are younger than patients with wild-type IDH1 gene 22,23 . Those results are similar to the findings of our study where we too found that patients with IDH1 $1^{\text {mut }}$ were younger than patients with IDH1 $1^{\text {wt }}(p<.05)$. We also found that patients in IDH $1^{\text {mut }}$ group were more likely to have a low-grade gliomas (grade II) compared with IDH'wt group. These findings replicate those of the above-mentioned studies.

The IDH1 gene mutation status is important prognostic marker in patients with diffuse gliomas. Yan et al. revealed that both IDH1 and IDH2 mutations were associated with better survival for patients with diffuse brain gliomas ${ }^{11}$. Moreover, Tabouret et al. demonstrated that IDHmut subgroup has significantly higher survival than IDH' subgroup for anaplastic astrocytoma and glioblastoma ${ }^{21}$. These results indicate that gliomas with $\mathrm{IDH}^{\text {mut }}$ are heterogeneous subgroup in diffuse brain gliomas. However, the mechanism by which IDH1 gene mutation affects survival time is still unclear. One of the possible mechanisms is that gliomas which arise from a IDH1 gene mutation require more time to convert into more aggressive glioblastomas and this might explain of why patients with IDH1 gene mutation have better survival. The majority of primary glioblastomas occur without IDH1 mutation in older patients, whereas secondary glioblastomas which arise from low grade gliomas often occur with IDH1 mutation in younger patients ${ }^{12}$.
The IDH1 protein is a cytosolic soluble enzyme which is encoded by IDH1 gene on chromosome 2 and catalyze the oxidative decarboxylation of isocitrate to 2-oxoglutarate ${ }^{24}$. The IDH1 protein is involved in various type of intracellular processes, such as cellular metabolism, epigenetic regulation and DNA damage ${ }^{25}$. Different types of IDH1 gene mutations at codon R132 have been identified such as c.395G>A (p.R132H) mutation accounts for about 93\%, c.394C>T (p.R132C) for $4 \%$, c.394C >A (p.R132S) for $1.5 \%$ in the different types of gliomas ${ }^{26,27}$. The point mutation affects the highly-conserved arginine residue at codon 132 (R132) in IDH1 gene are detected in $70 \%$ of low-grade gliomas such as oligodendrioglioma, oligoastrocytomas as well as secondary glioblastomas ${ }^{11}$. But primary glioblastomas and other systemic cancers very rarely contain IDH1 gene mutation.

Mutations at the codon 132 of IDH1 gene causes loss of its regular function and promotes tumorigenesis including diffuse brain gliomas ${ }^{28,29}$. Mutated IDH1 enzyme will produce 2-hydroxyglutarate which is considered oncometabolite and it may affect DNA methylation ${ }^{30}$. The recent genome-wide analysis demonstrated that IDH1 gene mutation is observed in greater than $80 \%$ of low-grade gliomas or secondary glioblastomas ${ }^{11,25,31}$. Therefore, IDH1 gene mutation might be key regulator in development of diffuse brain gliomas such as those evaluated in this study.

Our study has some limitations. We had relatively small sample size compared with other similar studies ${ }^{11,23}$. We used IHC staining method to reveal IDH1 gene mutation and this method has less sensitivity and specificity than a sequencing method. Also, several different types of IDH1 gene mutations at the codon 132 including R132H, R132S and R132C could be exist ${ }^{18}$. We only identified IDH1 mutation $\mathrm{R} 132 \mathrm{H}$ in this study. Therefore, future confirmative studies which have larger numbers of patients and utilize sequencing methods are needed to validate these results.

The main conclusions of this study are as follows: 1) IDH1 mutation is independent predictor of long-term prognosis in patients with diffuse brain gliomas, 2) IDH1 mutation is associated with better survival compared with wild-type IDH1.

\section{Conflict of interest}

The authors state no conflict of interest. 


\section{Acknowledgements}

This study was conducted by National Center for Pathology, Department of Pathology, MNUMS, on tissue obtained by the Department of Neurosurgery, The Third State Central Hospital. We acknowledge our sincerest gratitude for them.

\section{References}

1. Louis DN, Perry A, Reifenberger $G$, von Deimling A, Figarella-Branger D, Cavenee WK, et al. The 2016 World Health Organization Classification of Tumors of the Central Nervous System: a summary. Acta Neuropathol 2016; 131: 803-20.

2. Burger PC, Scheithauer BW, Paulus W, Szymas J, Giannini C, Kleihues P. Pathology and genetics of tumours of the nervous system. Lyon, France: IARC; 2000.

3. Wen PY, Kesari S. Malignant gliomas in adults. N Engl J Med 2008; 359: 492-507.

4. Cancer Genome Atlas Research Network. Comprehensive genomic characterization defines human glioblastoma genes and core pathways. Nature 2008; 455: 1061-8.

5. Li J, Yen C, Liaw D, Podsypanina K, Bose S, Wang SI, et al. PTEN, a putative protein tyrosine phosphatase gene mutated in human brain, breast, and prostate cancer. Science 1997; 275: 1943-7.

6. Nigro JM, Baker SJ, Preisinger AC, Jessup JM, Hostetter $R$, Cleary $\mathrm{K}$, et al. Mutations in the $\mathrm{p} 53$ gene occur in diverse human tumour types. Nature 1989; 342: 705-8.

7. Wong AJ, Bigner SH, Bigner DD, Kinzler KW, Hamilton SR and Vogelstein B. Increased expression of the epidermal growth factor receptor gene in malignant gliomas is invariably associated with gene amplification. Proc Natl Acad Sci U S A. 1987; 84: 6899-903.

8. Furnari FB, Fenton T, Bachoo RM, Mukasa A, Stommel JM, Stegh A, et al. Malignant astrocytic glioma: genetics, biology, and paths to treatment. Genes Dev 2007; 21: 2683-710.

9. Ohgaki $H$, Kleihues P. Genetic pathways to primary and secondary glioblastoma. Am J Pathol 2007; 170: 1445-53.

10. Weber RG, Sabel M, Reifenberger J, Sommer C, Oberstrass $J$, Reifenberger $G$, et al. Characterization of genomic alterations associated with glioma progression by comparative genomic hybridization. Oncogene 1996; 13:
983-94.

11. Yan $H$, Parsons DW, Jin G, McLendon R, Rasheed BA, Yuan W, et al. IDH1 and IDH2 mutations in gliomas. N Engl I Med 2009; 360: 765-73.

12. Ohgaki $H$, Kleihues $P$. The definition of primary and secondary glioblastoma. Clin Cancer Res 2013; 19: 764-72.

13. Felsberg J, Wolter M, Seul H, Friedensdorf B, Goppert M, Sabel MC, et al. Rapid and sensitive assessment of the IDH1 and IDH2 mutation status in cerebral gliomas based on DNA pyrosequencing. Acta Neuropathol 2010; 119: 501-7.

14. Kato $Y$, Jin $G$, Kuan $C T$, Mclendon RE, Yan H, Bigner DD. A monoclonal antibody IMab-1 specifically recognizes IDH1R132H, the most common glioma-derived mutation. Biochem Biophys Res Commun 2009; 390: 547-51.

15. Hartmann C, Meyer J, Balss J, Capper D, Mueller W, Christians $\mathrm{A}$, et al. Type and frequency of IDH1 and IDH2 mutations are related to astrocytic and oligodendroglial differentiation and age: a study of 1,010 diffuse gliomas. Acta Neuropathol 2009; 118: 469-74.

16. Capper D, Weissert S, Balss J, Habel A, Meyer J, Jager D, et al. Characterization of R132H mutation-specific IDH1 antibody binding in brain tumors. Brain Pathol 2010; 20: 245-54.

17. Capper D, Zentgraf H, Balss J, Hartmann C, von Deimling A. Monoclonal antibody specific for IDH1 R132H mutation. Acta Neuropathol 2009; 118: 599-601.

18. Mellai M, Piazzi A, Caldera V, Monzeglio O, Cassoni P, Valente G, et al. IDH1 and IDH2 mutations, immunohistochemistry and associations in a series of brain tumors. J Neurooncol 2011; 105: 345-57.

19. Enkhee O, Bolortuya D, Tuul B and Bayarmaa. Prognostic Value of Immunohistochemical Biomarkers in Patients with Astrocytic Brain Tumor. Cent Asian J Med Sci 2018; 4: 211 9. https://doi.org/10.24079/CAJMS.2018.09.007

20. National Institute of Health. IDH1 isocitrate dehydrogenase $(\operatorname{NADP}(+)) 1$, cytosolic [Homo sapiens (human)]. 2018.

21. Molenaar RJ, Maciejewski JP, Wilmink JW, van Noorden CJF. Wild-type and mutated IDH1/2 enzymes and therapy responses. Oncogene 2018; 37: 1949-60.

22. Balss J, Meyer J, Mueller W, Korshunov A, Hartmann C, von Deimling A. Analysis of the IDH1 codon 132 mutation in brain tumors. Acta Neuropathol 2008; 116: 597-602.

23. Deimling A, Korshunov A, Hartmann C. The next generation 
of glioma biomarkers: MGMT methylation, BRAF fusions and IDH1 mutations. Brain Pathol 2011; 21: 74-87.

24. Yang H, Ye D, Guan KL, Xiong Y. IDH1 and IDH2 mutations in tumorigenesis: mechanistic insights and clinical perspectives. Clin Cancer Res 2012; 18: 5562-71.

25. Dang L, Yen K, Attar EC. IDH mutations in cancer and progress toward development of targeted therapeutics. Ann Oncol 2016; 27: 599-608.

26. Ward PS, Cross JR, Lu C, Weigert O, Abel-Wahab O, Levine RL, et al. Identification of additional IDH mutations associated with oncometabolite R(-)-2-hydroxyglutarate production. Oncogene 2012; 31: 2491-8.

27. Parsons DW, Jones S, Zhang X, Lin JC, Leary RJ, Angenendt $P$, et al. An integrated genomic analysis of human glioblastoma multiforme. Science 2008; 321: 1807-12.
28. Labussiere M, Sanson M, Idbaih A, Delattre JY. IDH1 gene mutations: a new paradigm in glioma prognosis and therapy? Oncologist 2010; 15: 196-9.

29. Lv S, Teugels E, Sadones J, Quartier E, Huylebrouck M, DU Four $\mathrm{S}$, et al. Correlation between IDH1 gene mutation status and survival of patients treated for recurrent glioma. Anticancer Res 2011; 31: 4457-63.

30. Tabouret E, Nguyen AT, Dehais C, Carpentier C, Ducray F, Idbaih A, et al. Prognostic impact of the 2016 WHO classification of diffuse gliomas in the French POLA cohort. Acta Neuropathol 2016; 132: 625-34.

31. Akagi Y, Yoshimoto K, Hata N, Kug D, Hatae R, Amemiya T, et al. Reclassification of 400 consecutive glioma cases based on the revised 2016 WHO classification. Brain Tumor Pathol 2018; 35: 81-9. 